SISSA $32 / 2010 / \mathrm{EP}$

\title{
On Leptonic Unitary Triangles and Boomerangs
}

\author{
Alexander Dueck ${ }^{a *}, \quad$ Serguey T. Petcov ${ }^{b \dagger}, \quad$ Werner Rodejohann ${ }^{a \ddagger}$ \\ ${ }^{a}$ Max-Planck-Institut für Kernphysik, \\ Postfach 103980, D-69029 Heidelberg, Germany \\ ${ }^{b}$ SISSA and INFN - Sezione di Trieste, \\ Via Bonomea 265, I-34136 Trieste, Italy, \\ and \\ IPMU, University of Tokyo, Tokyo, Japan
}

\begin{abstract}
We review the idea of leptonic unitary triangles and extend the concept of the recently proposed unitary boomerangs to the lepton sector. Using a convenient parameterization of the lepton mixing, we provide approximate expressions for the side lengths and the angles of the six different triangles and give examples of leptonic unitary boomerangs. Possible applications of the leptonic unitary boomerangs are also briefly discussed.
\end{abstract}

*email: alexander. dueck@mpi-hd.mpg.de

${ }^{\dagger}$ Also at: INRNE, Bulgarian Academy of Sciences, 1784 Sofia, Bulgaria.

${ }^{\ddagger}$ email: werner.rodejohann@mpi-hd.mpg.de 
The leptonic, or Pontecorvo-Maki-Nakagawa-Sakata (PMNS), mixing matrix $U$ can be written as

$$
U=\left(\begin{array}{ccc}
c_{12} c_{13} & s_{12} c_{13} & s_{13} e^{-i \delta} \\
-s_{12} c_{23}-c_{12} s_{23} s_{13} e^{i \delta} & c_{12} c_{23}-s_{12} s_{23} s_{13} e^{i \delta} & s_{23} c_{13} \\
s_{12} s_{23}-c_{12} c_{23} s_{13} e^{i \delta} & -c_{12} s_{23}-s_{12} c_{23} s_{13} e^{i \delta} & c_{23} c_{13}
\end{array}\right) P
$$

where $c_{i j}=\cos \theta_{i j}, s_{i j}=\sin \theta_{i j}$ and $\delta$ is the unknown Dirac CP-violating phase. The two equally unknown Majorana phases [1,2] appear in $P=\operatorname{diag}\left(1, e^{i \phi_{2}}, e^{i \phi_{3}}\right)$. We will focus on the implications of $\delta$ in this letter. Namely, we discuss some aspects of unitarity of $U$ and Dirac-like CP violation in the form of unitarity triangles and boomerangs [3-5].

In the standard parametrization given above, $U$ is obtained by three consecutive rotations:

$$
\begin{gathered}
U=R_{23}\left(\theta_{23}\right) \tilde{R}_{13}\left(\theta_{13} ; \delta\right) R_{12}\left(\theta_{12}\right) \text {, where e.g. } \\
R_{12}\left(\theta_{12}\right)=\left(\begin{array}{ccc}
c_{12} & s_{12} & 0 \\
-s_{12} & c_{12} & 0 \\
0 & 0 & 1
\end{array}\right), \quad \tilde{R}_{13}\left(\theta_{13} ; \delta\right)=\left(\begin{array}{ccc}
c_{13} & 0 & s_{13} e^{-i \delta} \\
0 & 1 & 0 \\
-s_{13} e^{i \delta} & 0 & c_{13}
\end{array}\right)
\end{gathered}
$$

One notes that at zeroth order lepton mixing is well described (see Table 1) by tribimaximal mixing (TBM) 6

$$
U_{\mathrm{TBM}}=\left(\begin{array}{ccc}
\sqrt{\frac{2}{3}} & \sqrt{\frac{1}{3}} & 0 \\
-\sqrt{\frac{1}{6}} & \sqrt{\frac{1}{3}} & \sqrt{\frac{1}{2}} \\
\sqrt{\frac{1}{6}} & -\sqrt{\frac{1}{3}} & \sqrt{\frac{1}{2}}
\end{array}\right),
$$

or $U_{\mathrm{TBM}}=R_{23}(\pi / 4) R_{12}\left(\theta_{\mathrm{TBM}}\right)$, where $\sin ^{2} \theta_{\mathrm{TBM}}=\frac{1}{3}$. Accepting this rather economic scheme as the zeroth order description, makes possible to parameterize the PMNS matrix around $U_{\mathrm{TBM}}$, i.e. [7]

$$
U=R_{23}(\pi / 4) U_{\epsilon} R_{12}\left(\theta_{\mathrm{TBM}}\right), \text { where } U_{\epsilon}=R_{23}\left(\epsilon_{23}\right) \tilde{R}_{13}\left(\epsilon_{13} ; \delta\right) R_{12}\left(\epsilon_{12}\right) .
$$

The commonly used neutrino mixing observables are then obtained as zeroth order terms given by their TBM-values, and corrections in terms of the small $\epsilon_{i j}$ :

$$
\begin{aligned}
\sin ^{2} \theta_{12} & =\frac{1}{3}\left(\cos \epsilon_{12}+\sqrt{2} \sin \epsilon_{12}\right)^{2} \simeq \frac{1}{3}\left(1+2 \sqrt{2} \epsilon_{12}+\epsilon_{12}^{2}\right), \\
\sin ^{2} \theta_{23} & =\frac{1}{2}\left(1+\sin 2 \epsilon_{23}\right) \simeq \frac{1}{2}\left(1+2 \epsilon_{23}\right), \\
U_{e 3} & =\sin \epsilon_{13} e^{-i \delta}, \\
J_{\mathrm{CP}} & =\frac{1}{24}\left(2 \sqrt{2} \cos 2 \epsilon_{12}+\sin 2 \epsilon_{12}\right) \cos 2 \epsilon_{23} \sin 2 \epsilon_{13} \cos \epsilon_{13} \sin \delta \\
& \simeq\left(1+\frac{\epsilon_{12}}{\sqrt{2}}\right) \frac{\epsilon_{13}}{3 \sqrt{2}} \sin \delta .
\end{aligned}
$$




\begin{tabular}{c|ccc}
\hline \hline & & & \\
Parameter & best-fit & & \\
& & $2 \sigma$ & $3 \sigma$ \\
\hline & & & \\
$\sin ^{2} \theta_{12}$ & $0.318_{-0.016}^{+0.019}$ & $0.29-0.36$ & $0.27-0.38$ \\
$\sin ^{2} \theta_{23}$ & $0.500_{-0.060}^{+0.070}$ & $0.39-0.63$ & $0.36-0.67$ \\
$\sin ^{2} \theta_{13}$ & $0.013_{-0.009}^{+0.013}$ & $\leq 0.039$ & $\leq 0.053$ \\
\hline \hline
\end{tabular}

Table 1: Mixing angles and their best-fit values, $1 \sigma, 2 \sigma$ and $3 \sigma$ ranges [12].

We have expanded up to second order in the small parameters $\epsilon_{i j}$ and in the last line have given the usual Jarlskog invariant,

$$
J_{\mathrm{CP}}=\operatorname{Im}\left\{U_{e 1}^{*} U_{\mu 3}^{*} U_{e 3} U_{\mu 1}\right\}=\frac{1}{8} \sin 2 \theta_{12} \sin 2 \theta_{23} \sin 2 \theta_{13} \cos \theta_{13} \sin \delta,
$$

which describes leptonic Dirac-like CP violation. The advantage of the parameterization in Eq. (4) is that each $\theta_{i j}$ is corrected from its TBM-value by only one of the $\epsilon_{i j}$.

The angles in Table 1 are related to the elements of the PMNS matrix in the following way:

$$
\begin{gathered}
\sin ^{2} \theta_{13}=\left|U_{e 3}\right|^{2}, \quad \sin ^{2} \theta_{23}=\frac{\left|U_{\mu 3}\right|^{2}}{1-\left|U_{e 3}\right|^{2}}, \quad \cos ^{2} \theta_{23}=\frac{\left|U_{\tau 3}\right|^{2}}{1-\left|U_{e 3}\right|^{2}}, \\
\cos ^{2} \theta_{12}=\frac{\left|U_{e 1}\right|^{2}}{1-\left|U_{e 3}\right|^{2}}, \quad \sin ^{2} \theta_{12}=\frac{\left|U_{e 2}\right|^{2}}{1-\left|U_{e 3}\right|^{2}} .
\end{gathered}
$$

Information about the elements $\left|U_{e 1}\right|^{2},\left|U_{e 2}\right|^{2}$ and $\left|U_{e 3}\right|^{2}$ is obtained in the experiments with solar neutrinos and reactor antineutrinos. The experiments with atmospheric neutrinos provide data on $\left|U_{\mu 3}\right|^{2},\left|U_{\tau 3}\right|^{2}$ and $\left|U_{e 3}\right|^{2}$. These elements can be measured also in long baseline experiments with accelerator $\nu_{\mu}$ and $\bar{\nu}_{\mu}$ (MINOS, T2K, NO $\nu \mathrm{A}$, etc.). Some of these experiments will use very intense neutrino beams and will search for $\mathrm{CP}$ violating effects in neutrino oscillations. The magnitude of these effects is determined by [8] the rephasing invariant $J_{\mathrm{CP}}$, associated with the Dirac phase $\delta$.

The PMNS matrix is unitary, $U U^{\dagger}=U^{\dagger} U=\mathbb{1}$, and the six off-diagonal entries of $U U^{\dagger}=\mathbb{1}$ and $U^{\dagger} U=\mathbb{1}$ define six unitary triangles (properties of leptonic unitary triangles have been studied e.g. in Refs. 911]). Three stem from the conditions $U_{\alpha i}^{*} U_{\beta i}=0$ (the column-, or " $\alpha \beta$-triangles"), and the other three from $U_{\alpha i}^{*} U_{\alpha j}=0$ (the row-, or "ij-triangles"). To be more concrete:

$$
\begin{array}{ll}
\underline{e \mu}: & U_{e 1}^{*} U_{\mu 1}+U_{e 2}^{*} U_{\mu 2}+U_{e 3}^{*} U_{\mu 3}=0, \\
\underline{e \tau}: & U_{e 1}^{*} U_{\tau 1}+U_{e 2}^{*} U_{\tau 2}+U_{e 3}^{*} U_{\tau 3}=0, \\
\underline{\mu \tau}: & U_{\mu 1}^{*} U_{\tau 1}+U_{\mu 2}^{*} U_{\tau 2}+U_{\mu 3}^{*} U_{\tau 3}=0, \\
\underline{12}: & U_{e 1}^{*} U_{e 2}+U_{\mu 1}^{*} U_{\mu 2}+U_{\tau 1}^{*} U_{\tau 2}=0, \\
\underline{13}: & U_{e 1}^{*} U_{e 3}+U_{\mu 1}^{*} U_{\mu 3}+U_{\tau 1}^{*} U_{\tau 3}=0, \\
\underline{23}: & U_{e 2}^{*} U_{e 3}+U_{\mu 2}^{*} U_{\mu 3}+U_{\tau 2}^{*} U_{\tau 3}=0 .
\end{array}
$$


All six triangles have a common area of $A=\frac{1}{2} J_{\mathrm{CP}}$. If we rephase the rows of the PMNS matrix via $U_{\alpha i} \rightarrow U_{\alpha i} e^{i \phi_{i}}$, then the $\underline{\alpha \beta}$-triangles are invariant, whereas the $\underline{i j \text {-triangles are }}$ modified:

$$
U_{\alpha i}^{*} U_{\alpha j}=0 \rightarrow U_{\alpha i}^{*} U_{\alpha j} e^{i\left(\phi_{j}-\phi_{i}\right)}=0 .
$$

The $i j$-triangles are rotated in the complex plane. This shows that the Majorana phases influence the rotation of the $i j$-triangles and hence the $i j$-triangles are in principle probing them [10]: with a suitable phase rotation one can always arrange one side of the $\alpha \beta$ triangles to lie on the $x$ - or $y$-axis. If neutrinos are Majorana particles and the Majorana $\mathrm{CP}$ phases do not take on $\mathrm{CP}$ conserving values (i.e., $0 \bmod \pi / 2$ ) then this is not possible

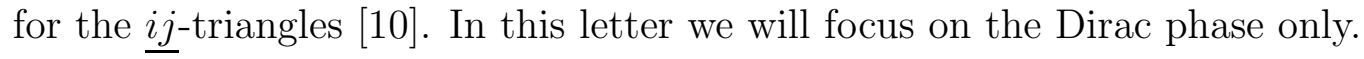

A triangle is defined by, e.g., two side lengths and one angle, which are three parameters, one short of the four parameters, $\theta_{12}, \theta_{13}, \theta_{23}$ and $\delta$, of the PMNS matrix, which enter into the expression for $J_{\mathrm{CP}}$ and are associated with the Dirac-like $\mathrm{CP}$ violation in the lepton sector. However, as two triangles share one common angle, it is possible to form an object called "boomerang" from two triangles [3-5]. The boomerang is described by four parameters. We will illustrate leptonic unitary boomerangs in the following, using the convenient parameterization from Eq. (4) of the PMNS matrix around the tri-bimaximal mixing.

The approximate form of the PMNS matrix,

$$
U=\mathcal{O}\left(\begin{array}{ccc}
1 & 1 & \lambda^{n} \\
1 & 1 & 1 \\
1 & 1 & 1
\end{array}\right)
$$

where $\lambda$ can be taken as the sine of the Cabibbo angle and the exponent $n \geq 1$ is currently unknown, implies that the $\underline{12}$ - and $\mu \tau$-triangles have sides of similar magnitude regardless of the value of $\left|U_{e 3}\right|$. To give a more precise estimate of the side lengths, we use now $\theta_{23}=\pi / 4, \theta_{12}=\theta_{\mathrm{TBM}}$, and keep only non-zero $\theta_{13}=\epsilon_{13}$. The side lengths of the six triangles are:

$$
\begin{array}{lc}
\underline{e \mu}: & \frac{1}{3}, \frac{1}{3}, \frac{\epsilon_{13}}{\sqrt{2}}, \\
\underline{e \tau}: & \frac{1}{3}, \frac{1}{3}, \frac{\epsilon_{13}}{\sqrt{2}}, \\
\underline{\mu \tau}: & \frac{1}{3}, \frac{1}{6}, \frac{1}{2}, \\
\underline{12}: & \frac{1}{3 \sqrt{2}}, \frac{\sqrt{2}}{3}, \frac{1}{3 \sqrt{2}}, \\
\underline{13}: & \frac{1}{2 \sqrt{3}}, \sqrt{\frac{2}{3}} \epsilon_{13}, \frac{1}{2 \sqrt{3}}, \\
\underline{23}: & \frac{1}{\sqrt{6}}, \frac{\epsilon_{13}}{\sqrt{3}}, \frac{1}{\sqrt{6}} .
\end{array}
$$

Hence, the maximal difference between two sides amounts to a factor $\simeq 3(\simeq 2)$ for the $\mu \tau$-(12-)triangle. For the maximal allowed value of $\epsilon_{13}^{\max }=0.23$ the maximal difference for the other four triangles (from top to bottom) is about 2, 2, 1.5 and 3. Taking the best-fit 


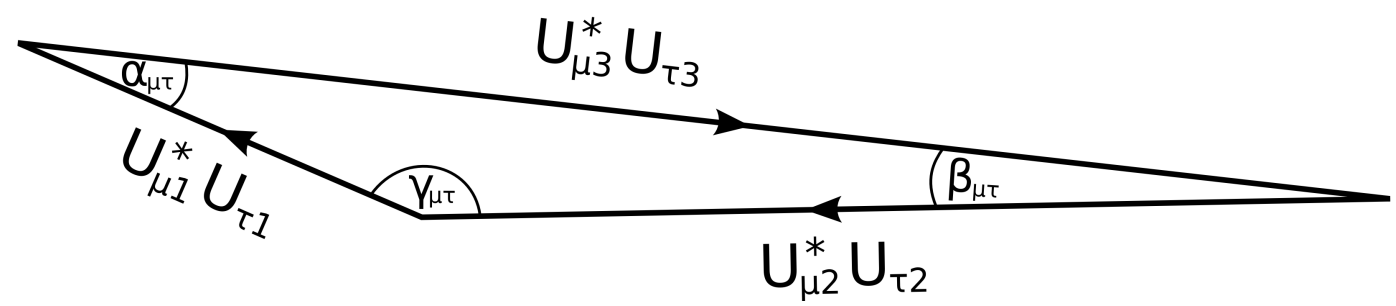

Figure 1: The $\mu \tau$-triangle with best-fit values for the mixing parameters (see Tab. 1) and $\delta=\pi / 2$. The angles are $\alpha_{\mu \tau} \simeq 19.0^{\circ}, \beta_{\mu \tau} \simeq 8.9^{\circ}$, and $\gamma_{\mu \tau} \simeq 152.1^{\circ}$, the side lengths $\left|U_{\mu 2}^{*} U_{\tau 2}\right| \simeq 0.34,\left|U_{\mu 1}^{*} U_{\tau 1}\right| \simeq 0.16$, and $\left|U_{\mu 3}^{*} U_{\tau 3}\right| \simeq 0.49$. The area is $A_{\underline{\mu \tau}}=1 / 2\left|U_{\mu 2}^{*} U_{\tau 2}\right|\left|U_{\mu 3}^{*} U_{\tau 3}\right| \sin \beta_{\mu \tau} \simeq 0.013$.

value of $\epsilon_{13}^{\text {best-fit }}=0.11$ yields values of about $4,4,3$ and 6 . We will focus here for the sake of illustration on the 12 - and $\mu \tau$-triangles and their resulting boomerang, and delegate the formulae for the remaining four triangles to the Appendix.

Consider first the $\underline{\mu \tau}$-triangle (see Fig. 10): the lengths of the sides are

$$
\begin{aligned}
\left|U_{\mu 2}^{*} U_{\tau 2}\right| & \simeq \frac{1}{3}\left(1-\sqrt{2} \epsilon_{12}-\frac{1}{2} \epsilon_{12}^{2}-2 \epsilon_{23}^{2}-2 \sqrt{2} \epsilon_{13} \epsilon_{23} \cos \delta-\frac{1}{2} \epsilon_{13}^{2} \cos 2 \delta\right) \\
\left|U_{\mu 1}^{*} U_{\tau 1}\right| & \simeq \frac{1}{6}\left(1+2 \sqrt{2} \epsilon_{12}+\epsilon_{12}^{2}-2 \epsilon_{23}^{2}+4 \sqrt{2} \epsilon_{13} \epsilon_{23} \cos \delta-2 \epsilon_{13}^{2} \cos 2 \delta\right) \\
\left|U_{\mu 3}^{*} U_{\tau 3}\right| & \simeq \frac{1}{2}\left(1-\epsilon_{13}^{2}-2 \epsilon_{23}^{2}\right) .
\end{aligned}
$$

The angles are

$$
\begin{aligned}
& \alpha_{\mu \tau}=\arg \left\{-\frac{U_{\mu 3}^{*} U_{\tau 3}}{U_{\mu 1}^{*} U_{\tau 1}}\right\} \simeq\left(2 \sqrt{2} \epsilon_{13}-6 \epsilon_{12} \epsilon_{13}\right) \sin \delta, \\
& \beta_{\mu \tau}=\arg \left\{-\frac{U_{\mu 2}^{*} U_{\tau 2}}{U_{\mu 3}^{*} U_{\tau 3}}\right\} \simeq\left(\sqrt{2} \epsilon_{13}+3 \epsilon_{12} \epsilon_{13}\right) \sin \delta, \\
& \gamma_{\mu \tau}=\arg \left\{-\frac{U_{\mu 1}^{*} U_{\tau 1}}{U_{\mu 2}^{*} U_{\tau 2}}\right\} \simeq\left(-3 \sqrt{2} \epsilon_{13}+3 \epsilon_{12} \epsilon_{13}\right) \sin \delta .
\end{aligned}
$$

The definitions of the angles are such that the sides are ordered as written in Eqs. (12 17) and $\alpha$ is the angle between the third and the first sides, $\beta$ between the second and third, and $\gamma$ between the first and second. With these definitions all the angles can be positive or negative and their sum can be equal either to $\pi$ or to $(-\pi): \alpha_{\mu \tau}+\beta_{\mu \tau}+\gamma_{\mu \tau}= \pm \pi$ | If, for instance, we use the exact expressions in Eqs. (29 31), the best-fit values given in Table 1 and $\delta=\pi / 2$, we get $\alpha_{\mu \tau} \simeq 19.0^{\circ}, \beta_{\mu \tau} \simeq 8.9^{\circ}, \gamma_{\mu \tau} \simeq 152.1^{\circ}$, and $\alpha_{\mu \tau}+\beta_{\mu \tau}+\gamma_{\mu \tau}=$ $\pi$. For $\delta=-\pi / 2$, however, we find $\alpha_{\mu \tau} \simeq-19.0^{\circ}, \beta_{\mu \tau} \simeq-8.9^{\circ}, \gamma_{\mu \tau} \simeq-152.1^{\circ}$, and

\footnotetext{
IThis can be shown for instance by multiplying in $\alpha_{\mu \tau}=\arg \left\{-\frac{U_{\mu 3}^{*} U_{\tau 3}}{U_{\mu 1}^{*} U_{\tau 1}}\right\}$ the denominator and numerator with $U_{\mu 2}^{*} U_{\tau 2}$, performing simple manipulations and noting that $\arg \{-1\}= \pm \pi$.
} 


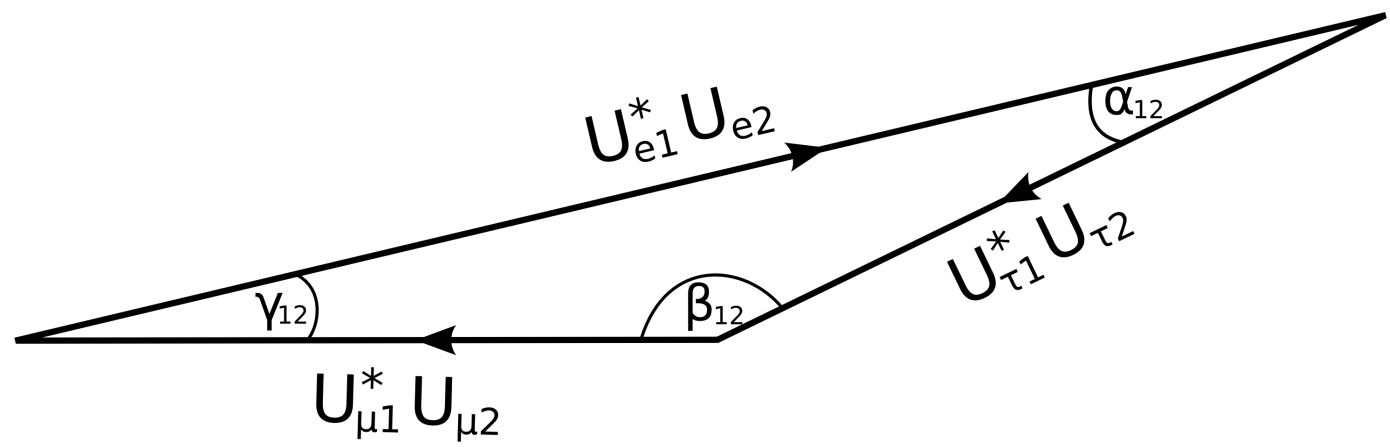

Figure 2: The 12-triangle with best-fit values for the mixing parameters and $\delta=\pi / 2$. The angles are $\alpha_{12} \simeq 13.9^{\circ}, \beta_{12} \simeq 152.1^{\circ}$, and $\gamma_{12} \simeq 13.9^{\circ}$, the side lengths $\left|U_{\mu 1}^{*} U_{\mu 2}\right| \simeq 0.24$, $\left|U_{e 1}^{*} U_{e 2}\right| \simeq 0.46$, and $\left|U_{\tau 1}^{*} U_{\tau 2}\right| \simeq 0.24$. The area is $A_{\underline{12}}=1 / 2\left|U_{\mu 1}^{*} U_{\mu 2}\right|\left|U_{e 1}^{*} U_{e 2}\right| \sin \gamma_{12} \simeq$ 0.013. The $\underline{\mu \tau}$-triangle and the $\underline{12}$-triangle (Fig. 1) have one common angle: $\gamma_{\mu \tau}=\beta_{12}$.

$\alpha_{\mu \tau}+\beta_{\mu \tau}+\gamma_{\mu \tau}=-\pi$. The approximate expressions for the angles that we give in this letter add up to zero. Depending on the signs and values of the $\epsilon_{i j}$ and of $\delta$, one has to add $\pi$ or $-\pi$ to one of the expressions. For instance, for the best-fit values and $\delta=\pi / 2$ $(-\pi / 2)$ one would have to add $\pi(-\pi)$ to $\gamma_{\mu \tau}$ in Eq. (31). For the angles of the 12 -triangle given below in Eqs. (32 34) one would have to add $\pi(-\pi)$ to $\beta_{12}$. Similar comments are valid for the angles of the other unitarity triangles we will consider in what follows. We show the unitary $\mu \tau$-triangle in Fig. 1 in the case of $\delta=\pi / 2$ and for the best-fit values from Table 1 .

As we see, in the case of the $\mu \tau$-triangle, the Dirac CP-violating phase $\delta$ does not coincide with any of the angles of the triangle. For some of the other triangles, however, the Dirac phase $\delta$ corresponds to certain angles: we have, for instance, $\delta \simeq \alpha_{e \mu}$ (see the Appendix). The 12-triangle side lengths are given by

$$
\begin{aligned}
\left|U_{\mu 1}^{*} U_{\mu 2}\right| & \simeq \frac{1}{3 \sqrt{2}}\left(1+\frac{1}{\sqrt{2}} \epsilon_{12}-2 \epsilon_{23}+\frac{1}{\sqrt{2}} \epsilon_{13} \cos \delta-2 \epsilon_{12}^{2}-\epsilon_{13}^{2}\left(1-\frac{9}{4} \sin ^{2} \delta\right)\right. \\
& \left.-\sqrt{2} \epsilon_{12} \epsilon_{23}-4 \epsilon_{12} \epsilon_{13} \cos \delta\right), \\
\left|U_{e 1}^{*} U_{e 2}\right| \simeq & \frac{\sqrt{2}}{3}\left(1+\frac{1}{\sqrt{2}} \epsilon_{12}-2 \epsilon_{12}^{2}-\epsilon_{13}^{2}\right), \\
\left|U_{\tau 1}^{*} U_{\tau 2}\right| \simeq & \frac{1}{3 \sqrt{2}}\left(1+\frac{1}{\sqrt{2}} \epsilon_{12}+2 \epsilon_{23}-\frac{1}{\sqrt{2}} \epsilon_{13} \cos \delta-2 \epsilon_{12}^{2}-\epsilon_{13}^{2}\left(1-\frac{9}{4} \sin ^{2} \delta\right)\right. \\
+ & \left.\sqrt{2} \epsilon_{12} \epsilon_{23}+4 \epsilon_{12} \epsilon_{13} \cos \delta\right),
\end{aligned}
$$

and the angles by 


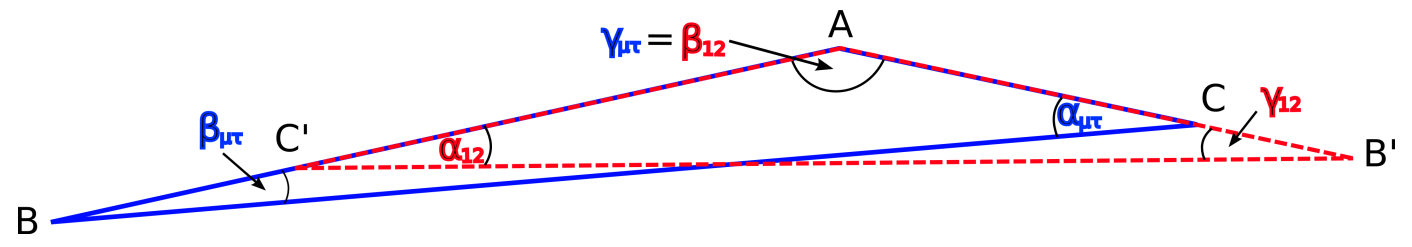

Figure 3: By overlapping the $\mu \tau$-triangle from Fig. 1 and the 12 -triangle from Fig. 2 at their common angle we get the unitary $\mu \tau$-12-boomerang.

$$
\begin{aligned}
& \alpha_{12}=\arg \left\{-\frac{U_{\tau 1}^{*} U_{\tau 2}}{U_{e 1}^{*} U_{e 2}}\right\} \simeq\left(\frac{3}{\sqrt{2}} \epsilon_{13}-\frac{3}{2} \epsilon_{12} \epsilon_{13}-3 \sqrt{2} \epsilon_{13} \epsilon_{23}\right) \sin \delta+\frac{3}{4} \epsilon_{13}^{2} \sin 2 \delta, \\
& \beta_{12}=\arg \left\{-\frac{U_{\mu 1}^{*} U_{\mu 2}}{U_{\tau 1}^{*} U_{\tau 2}}\right\} \simeq\left(-3 \sqrt{2} \epsilon_{13}+3 \epsilon_{12} \epsilon_{13}\right) \sin \delta, \\
& \gamma_{12}=\arg \left\{-\frac{U_{e 1}^{*} U_{e 2}}{U_{\mu 1}^{*} U_{\mu 2}}\right\} \simeq\left(\frac{3}{\sqrt{2}} \epsilon_{13}-\frac{3}{2} \epsilon_{12} \epsilon_{13}+3 \sqrt{2} \epsilon_{13} \epsilon_{23}\right) \sin \delta-\frac{3}{4} \epsilon_{13}^{2} \sin 2 \delta .
\end{aligned}
$$

Using again the best-fit values from Table 1 and $\delta=\pi / 2$ we show the unitary 12 -triangle in Fig. 2,

It is obvious from Eqs. (31) and (36) that $\gamma_{\mu \tau}=\beta_{12}$. This allows to "glue" two triangles together and form an object called boomerang [3]. The $\mu \tau$-12-boomerang is shown in Fig. 3. The side lengths between the points are: $\mathrm{AC}=\left|U_{\mu 1}^{*} \overline{U_{\tau 1}}\right| \simeq 0.16, \mathrm{AC}^{\prime}=\left|U_{\tau 1}^{*} U_{\tau 2}\right| \simeq$ $0.24, \mathrm{AB}=\left|U_{\mu 2}^{*} U_{\tau 2}\right| \simeq 0.34, \mathrm{AB}^{\prime}=\left|U_{\mu 1}^{*} U_{\mu 2}\right| \simeq 0.24, \mathrm{BC}=\left|U_{\mu 3}^{*} U_{\tau 3}\right| \simeq 0.49$ and $\mathrm{BC}^{\prime}$ $=\left|U_{e 1}^{*} U_{e 2}\right| \simeq 0.46$. Certain areas can be connected to the PMNS matrix elements and the Jarlskog invariant [5]. For instance,

$$
A_{\mathrm{ABB}^{\prime}}=\frac{1}{2} \frac{\left|U_{\mu 2}\right|}{\left|U_{\tau 1}\right|} J_{\mathrm{CP}} \simeq 0.019, \quad A_{\mathrm{ACC}^{\prime}}=\frac{1}{2} \frac{\left|U_{\tau 1}\right|}{\left|U_{\mu 2}\right|} J_{\mathrm{CP}} \simeq 0.009
$$

and thus $A_{\mathrm{ABB}^{\prime}} A_{\mathrm{ACC}^{\prime}}=\frac{1}{4} J_{\mathrm{CP}}^{2} \simeq 1.7 \cdot 10^{-4}$. If there was some form of new physics which leaves $\gamma_{\mu \tau}$ and $\beta_{12}$ unaffected, then one might probe its presence by checking whether the product of the above areas is indeed given by $\frac{1}{4} J_{\mathrm{CP}}^{2}$.

Unitary boomerangs are also a useful method to study the presence of new physics which one single unitary triangle might miss [5]. Consider, for instance, the existence of a light sterile neutrino (generalization to more sterile species is straightforward). There would

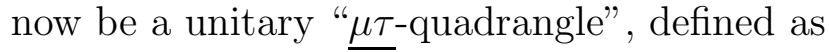

$$
U_{\mu 1}^{*} U_{\tau 1}+U_{\mu 2}^{*} U_{\tau 2}+U_{\mu 3}^{*} U_{\tau 3}+U_{\mu 4}^{*} U_{\tau 4}=0 .
$$

In case of $U_{\tau 4}=0$ ( or $U_{\mu 4}=0$ ), the experimental study of the $\mu \tau$-triangle would reveal no

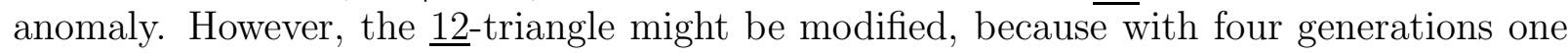
has now

$$
U_{e 1}^{*} U_{e 2}+U_{\mu 1}^{*} U_{\mu 2}+U_{\tau 1}^{*} U_{\tau 2}=-U_{s 1}^{*} U_{s 2}
$$


Unless $U_{s 1}=0$ or $U_{s 2}=0$, an inconsistency between the two triangles would appear and the $\underline{\mu \tau}$-12-boomerang could not be formed.

Unitary boomerangs are therefore a useful, illustrative and comprehensive method to study the consistence of the standard neutrino framework.

\section{Acknowledgments}

We would like to thank P. Frampton for discussions on unitary boomerangs. This work was supported by the ERC under the Starting Grant MANITOP and by the DFG in the Transregio 27 (A.D. and W.R.), as well as by World Premier International Research Center Initiative (WPI Initiative), MEXT, Japan, and the INFN program on "Astroparticle Physics" (S.T.P.).

\section{A Formulae for the Remaining Unitary Triangles}

In the following we give the side lengths and angles for the remaining four unitary triangles. We will expand the formulae in second order in the small $\epsilon_{i j}$. Recall that in the approximate expressions for the angles they add up to zero, and depending on the signs and values of the $\epsilon_{i j}$ and of $\delta$ one needs to add $\pm \pi$ for appropriate angles (see main text).

For the $\underline{e \mu}$-triangle one obtains the side lengths as

$$
\begin{aligned}
&\left|U_{e 2}^{*} U_{\mu 2}\right| \simeq \frac{1}{3}\left(1+\frac{\epsilon_{12}}{\sqrt{2}}-\epsilon_{23}-\frac{1}{2}\left(\sqrt{2} \epsilon_{13}+4 \epsilon_{12} \epsilon_{13}+\sqrt{2} \epsilon_{13} \epsilon_{23}\right) \cos \delta\right.\left.-2 \epsilon_{12}^{2}-\frac{\epsilon_{12} \epsilon_{23}}{\sqrt{2}}-\frac{\epsilon_{23}^{2}}{2}-\frac{\epsilon_{13}^{2}}{4}\left(2-\sin ^{2} \delta\right)\right) \\
&\left|U_{e 1}^{*} U_{\mu 1}\right| \simeq \frac{1}{3}\left(1+\frac{\epsilon_{12}}{\sqrt{2}}-\epsilon_{23}+\left(\sqrt{2} \epsilon_{13}-2 \epsilon_{12} \epsilon_{13}+\sqrt{2} \epsilon_{13} \epsilon_{23}\right) \cos \delta\right. \\
&\left.\quad-2 \epsilon_{12}^{2}-\frac{\epsilon_{12} \epsilon_{23}}{\sqrt{2}}-\frac{\epsilon_{23}^{2}}{2}-\frac{1}{2} \epsilon_{13}^{2} \cos 2 \delta\right), \\
&\left|U_{e 3}^{*} U_{\mu 3}\right| \simeq \frac{\epsilon_{13}}{\sqrt{2}}+\frac{\epsilon_{13} \epsilon_{23}}{\sqrt{2}}
\end{aligned}
$$

while the angles are

$$
\begin{aligned}
& \alpha_{e \mu}=\arg \left\{-\frac{U_{e 3}^{*} U_{\mu 3}}{U_{e 1}^{*} U_{\mu 1}}\right\} \simeq \delta-\left(\sqrt{2} \epsilon_{13}-\epsilon_{12} \epsilon_{13}\right) \sin \delta+\epsilon_{13}^{2} \sin 2 \delta, \\
& \beta_{e \mu}=\arg \left\{-\frac{U_{e 2}^{*} U_{\mu 2}}{U_{e 3}^{*} U_{\mu 3}}\right\} \simeq-\delta-\left(\frac{1}{\sqrt{2}} \epsilon_{13}-\frac{1}{2} \epsilon_{12} \epsilon_{13}\right) \sin \delta-\frac{1}{4} \epsilon_{13}^{2} \sin 2 \delta, \\
& \gamma_{e \mu}=\arg \left\{-\frac{U_{e 1}^{*} U_{\mu 1}}{U_{e 2}^{*} U_{\mu 2}}\right\} \simeq\left(\frac{3}{\sqrt{2}} \epsilon_{13}-\frac{3}{2} \epsilon_{12} \epsilon_{13}\right) \sin \delta-\frac{3}{4} \epsilon_{13}^{2} \sin 2 \delta .
\end{aligned}
$$

Note that in the limit of $\epsilon_{13}$ going to zero (which corresponds to $\left|U_{e 3}\right|$ going to zero and implies that $\mathrm{CP}$ violation in neutrino oscillations is absent), the exact expressions for $\alpha_{e \mu}$ 
and $\beta_{e \mu}$ are undefined, while the approximate expressions for these two angles are formally given by $\delta$ and $-\delta$, respectively. As $\delta$ is however unphysical when $\epsilon_{13}=0$, there is no inconsistency. Turning to the $\underline{e \tau}$-triangle, we find

$$
\begin{aligned}
&\left|U_{e 2}^{*} U_{\tau 2}\right| \simeq \frac{1}{3}( 1+\frac{1}{\sqrt{2}} \epsilon_{12}+\epsilon_{23}+\left(\frac{1}{\sqrt{2}} \epsilon_{13}+2 \epsilon_{12} \epsilon_{13}-\frac{1}{\sqrt{2}} \epsilon_{13} \epsilon_{23}\right) \cos \delta \\
&\left.-2 \epsilon_{12}^{2}-\frac{1}{2} \epsilon_{23}^{2}+\frac{1}{\sqrt{2}} \epsilon_{12} \epsilon_{23}-\frac{1}{4} \epsilon_{13}^{2}\left(2-\sin ^{2} \delta\right)\right), \\
&\left|U_{e 1}^{*} U_{\tau 1}\right| \simeq \frac{1}{3}\left(1+\frac{1}{\sqrt{2}} \epsilon_{12}+\epsilon_{23}+\left(-\sqrt{2} \epsilon_{13}+2 \epsilon_{12} \epsilon_{13}+\sqrt{2} \epsilon_{13} \epsilon_{23}\right) \cos \delta\right.\left.-2 \epsilon_{12}^{2}+\frac{1}{\sqrt{2}} \epsilon_{12} \epsilon_{23}-\frac{1}{2} \epsilon_{23}^{2}-\frac{1}{2} \epsilon_{13}^{2} \cos 2 \delta\right), \\
&\left|U_{e 3}^{*} U_{\tau 3}\right| \simeq \frac{1}{\sqrt{2}} \epsilon_{13}-\frac{1}{\sqrt{2}} \epsilon_{13} \epsilon_{23},
\end{aligned}
$$

and

$$
\begin{aligned}
& \alpha_{e \tau}=\arg \left\{-\frac{U_{e 3}^{*} U_{\tau 3}}{U_{e 1}^{*} U_{\tau 1}}\right\} \simeq \delta+\left(\sqrt{2} \epsilon_{13}-\epsilon_{12} \epsilon_{13}\right) \sin \delta+\epsilon_{13}^{2} \sin 2 \delta, \\
& \beta_{e \tau}=\arg \left\{-\frac{U_{e 2}^{*} U_{\tau 2}}{U_{e 3}^{*} U_{\tau 3}}\right\} \simeq-\delta+\left(\frac{1}{\sqrt{2}} \epsilon_{13}-\frac{1}{2} \epsilon_{12} \epsilon_{13}\right) \sin \delta-\frac{1}{4} \epsilon_{13}^{2} \sin 2 \delta, \\
& \gamma_{e \tau}=\arg \left\{-\frac{U_{e 1}^{*} U_{\tau 1}}{U_{e 2}^{*} U_{\tau 2}}\right\} \simeq\left(-\frac{3}{\sqrt{2}} \epsilon_{13}+\frac{3}{2} \epsilon_{12} \epsilon_{13}+3 \sqrt{2} \epsilon_{13} \epsilon_{23}\right) \sin \delta-\frac{3}{4} \epsilon_{13}^{2} \sin 2 \delta .
\end{aligned}
$$

For the 13-triangle we have

$$
\begin{aligned}
\left|U_{\mu 1}^{*} U_{\mu 3}\right| & \simeq \frac{1}{2 \sqrt{3}}\left(1+\sqrt{2} \epsilon_{12}+\sqrt{2} \epsilon_{13} \cos \delta-\frac{1}{2} \epsilon_{12}^{2}-2 \epsilon_{23}^{2}+\left(2 \sqrt{2} \epsilon_{13} \epsilon_{23}-\epsilon_{12} \epsilon_{13}\right) \cos \delta\right. \\
\left.\quad-\frac{1}{2} \epsilon_{13}^{2} \cos 2 \delta\right) & \\
\left|U_{e 1}^{*} U_{e 3}\right| & \simeq \sqrt{\frac{2}{3}} \epsilon_{13}-\frac{1}{\sqrt{3}} \epsilon_{12} \epsilon_{13} \\
\left|U_{\tau 1}^{*} U_{\tau 3}\right| & \simeq \frac{1}{2 \sqrt{3}}\left(1+\sqrt{2} \epsilon_{12}-\sqrt{2} \epsilon_{13} \cos \delta-\frac{1}{2} \epsilon_{12}^{2}-2 \epsilon_{23}^{2}+\left(\epsilon_{12} \epsilon_{13}+2 \sqrt{2} \epsilon_{13} \epsilon_{23}\right) \cos \delta\right. \\
- & \left.\frac{1}{2} \epsilon_{13}^{2} \cos 2 \delta\right)
\end{aligned}
$$

and

$$
\begin{aligned}
& \alpha_{13}=\arg \left\{-\frac{U_{\tau 1}^{*} U_{\tau 3}}{U_{e 1}^{*} U_{e 3}}\right\} \simeq \delta+\left(\sqrt{2} \epsilon_{13}-\epsilon_{12} \epsilon_{13}\right) \sin \delta+\epsilon_{13}^{2} \sin 2 \delta \\
& \beta_{13}=\arg \left\{-\frac{U_{\mu 1}^{*} U_{\mu 3}}{U_{\tau 1}^{*} U_{\tau 3}}\right\} \simeq\left(-2 \sqrt{2} \epsilon_{13}+6 \epsilon_{12} \epsilon_{13}\right) \sin \delta \\
& \gamma_{13}=\arg \left\{-\frac{U_{e 1}^{*} U_{e 3}}{U_{\mu 1}^{*} U_{\mu 3}}\right\} \simeq-\delta+\left(\sqrt{2} \epsilon_{13}-\epsilon_{12} \epsilon_{13}\right) \sin \delta-\epsilon_{13}^{2} \sin 2 \delta .
\end{aligned}
$$


Finally, we get for the $\underline{23}$-triangle

$$
\begin{aligned}
\left|U_{\mu 2}^{*} U_{\mu 3}\right| & \simeq \frac{1}{\sqrt{6}}\left(1-\frac{1}{\sqrt{2}} \epsilon_{12}-\frac{1}{\sqrt{2}} \epsilon_{13} \cos \delta-\frac{1}{2} \epsilon_{12}^{2}-2 \epsilon_{23}^{2}\right. \\
& \left.-\left(\epsilon_{12} \epsilon_{13}+\sqrt{2} \epsilon_{13} \epsilon_{23}\right) \cos \delta-\frac{1}{4} \epsilon_{13}^{2}\left(2-\sin ^{2} \delta\right)\right), \\
\left|U_{e 2}^{*} U_{e 3}\right| \simeq & \frac{1}{\sqrt{3}} \epsilon_{13}+\sqrt{\frac{2}{3}} \epsilon_{12} \epsilon_{13}, \\
\left|U_{\tau 2}^{*} U_{\tau 3}\right| \simeq & \frac{1}{\sqrt{6}}\left(1-\frac{1}{\sqrt{2}} \epsilon_{12}+\frac{1}{\sqrt{2}} \epsilon_{13} \cos \delta-\frac{1}{2} \epsilon_{12}^{2}-2 \epsilon_{23}^{2}\right. \\
& \left.+\left(\epsilon_{12} \epsilon_{13}-\sqrt{2} \epsilon_{13} \epsilon_{23}\right) \cos \delta-\frac{1}{4} \epsilon_{13}^{2}\left(2-\sin ^{2} \delta\right)\right),
\end{aligned}
$$

and for its angles

$$
\begin{aligned}
& \alpha_{23}=\arg \left\{-\frac{U_{\tau 2}^{*} U_{\tau 3}}{U_{e 2}^{*} U_{e 3}}\right\} \simeq \delta-\left(\frac{1}{\sqrt{2}} \epsilon_{13}-\frac{1}{2} \epsilon_{12} \epsilon_{13}\right) \sin \delta+\frac{1}{4} \epsilon_{13}^{2} \sin 2 \delta, \\
& \beta_{23}=\arg \left\{-\frac{U_{\mu 2}^{*} U_{\mu 3}}{U_{\tau 2}^{*} U_{\tau 3}}\right\} \simeq\left(\sqrt{2} \epsilon_{13}+3 \epsilon_{12} \epsilon_{13}\right) \sin \delta, \\
& \gamma_{23}=\arg \left\{-\frac{U_{e 2}^{*} U_{e 3}}{U_{\mu 2}^{*} U_{\mu 3}}\right\} \simeq-\delta-\left(\frac{1}{\sqrt{2}} \epsilon_{13}-\frac{1}{2} \epsilon_{12} \epsilon_{13}\right) \sin \delta-\frac{1}{4} \epsilon_{13}^{2} \sin 2 \delta .
\end{aligned}
$$

The following relations between the angles are found:

$$
\begin{gathered}
\alpha_{e \mu}=-\gamma_{13}, \beta_{e \mu}=\gamma_{23}, \gamma_{e \mu}=\gamma_{12}, \\
\alpha_{e \tau}=\alpha_{13}, \beta_{e \tau}=-\alpha_{23}, \gamma_{e \tau}=-\alpha_{12}, \\
\alpha_{\mu \tau}=-\beta_{13}, \beta_{\mu \tau}=\beta_{23}, \gamma_{\mu \tau}=\beta_{12} .
\end{gathered}
$$

There are only 9 different angles among the 18 in total. For each pair of identical angles one can overlap the longer side of one triangle with the shorter side of the other triangle or vice versa. There are therefore 18 possible unitary boomerangs [3].

\section{References}

[1] S. M. Bilenky, J. Hosek and S. T. Petcov, Phys. Lett. B 94, 495 (1980).

[2] J. Schechter and J. W. F. Valle, Phys. Rev. D 22, 2227 (1980); M. Doi, T. Kotani, H. Nishiura, K. Okuda and E. Takasugi, Phys. Lett. B 102, 323 (1981).

[3] P. H. Frampton and X. G. He, Phys. Lett. B 688, 67 (2010) arXiv:1003.0310 [hep-ph]].

[4] S. W. Li and B. Q. Ma, arXiv:1003.5854 [hep-ph].

[5] P. H. Frampton and X. G. He, arXiv:1004.3679 [hep-ph]. 
[6] P. F. Harrison, D. H. Perkins and W. G. Scott, Phys. Lett. B 530, 167 (2002) arXiv:hep-ph/0202074]; Phys. Lett. B 535, 163 (2002) arXiv:hep-ph/0203209; Z. Z. Xing, Phys. Lett. B 533, 85 (2002) arXiv:hep-ph/0204049]; X. G. He and A. Zee, Phys. Lett. B 560, 87 (2003) arXiv:hep-ph/0301092].

[7] S. Pakvasa, W. Rodejohann and T. J. Weiler, Phys. Rev. Lett. 100, 111801 (2008) arXiv:0711.0052 [hep-ph]].

[8] P. I. Krastev and S. T. Petcov, Phys. Lett. B 205, 84 (1988).

[9] Y. Farzan and A. Y. Smirnov, Phys. Rev. D 65, 113001 (2002) arXiv:hep-ph/0201105.

[10] J. A. Aguilar-Saavedra and G. C. Branco, Phys. Rev. D 62, 096009 (2000) arXiv:hep-ph/0007025].

[11] Z. Z. Xing and H. Zhang, Phys. Lett. B 618, 131 (2005) arXiv:hep-ph/0503118; J. D. Bjorken, P. F. Harrison and W. G. Scott, Phys. Rev. D 74, 073012 (2006) arXiv:hep-ph/0511201.

[12] T. Schwetz, M. A. Tortola and J. W. F. Valle, New J. Phys. 10, 113011 (2008) arXiv:0808.2016v3 [hep-ph]]. 